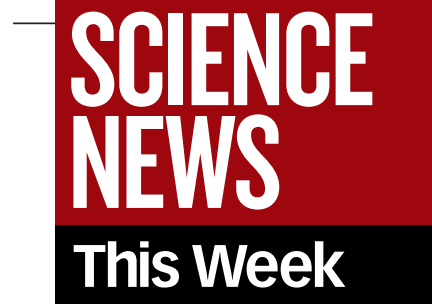

Hui, the crack "just sits there and opens up"a phenomenon known as blunting.

While materials investigators had previously noted blunting at crack tips in some soft solids, the DuPont-Cornell team has identified a new mechanism of crack propagation that incorporates blunting. "It's really a fundamental change in [comprehending] the way a crack runs in a material," Bennison says.

Kenneth R. Shull of Northwestern University in Evanston, Ill., agrees. "I really do think it is potentially one of the key ideas ... that's been largely missed before," he says.

In materials-speak, a tough substance is one that absorbs a lot of energy as it breaks apart. Consider the transparent polymer called plasticized polyvinyl butyral, or PVB, that DuPont makes to cement sheets of glass together in car windshields. PVB puzzled DuPont scientists, Bennison notes, because it was tougher than could be accounted for by the prevailing ideas of how materials fail. DuPont-Cornell team made the startling experimental observation that a slit the length of a paperclip in stretched PVB would open into a hole rather than tear the fabric further. Adding to this unexpected, macroscopic demonstration of crack blunting, computer simulations of fracturing in even more elastic materials pointed to blunting as a barrier to crack growth on a microscopic scale.
In studies that began in 1995, the

To understand what makes these materials give way when they finally do, the team found X-ray evidence that thousands of minuscule voids-the result of blunting of submicroscopic cracks-set the stage for the failure. The scientists contend also that stretching around blunted microcracks eventually stiffens the material to the point that it can't deform any more, leading to the massive breaking of chemical bonds that sums into ordinary fractures (SN: 1/4/O3, p. 3).

Manoj K. Chaudhury of Lehigh University in Bethlehem, Pa., calls the new work the first quantitative theory to show that blunting can account for the toughness of rubbery materials. Nonetheless, cautions Shull, many more experiments are needed to see if it's possible to poke holes in this new theory. -P. WEISS

\section{Out of China}

\section{SARS virus' genome hints at independent evolution}

The newly deciphered genome of the pathogen responsible for severe acute respiratory syndrome (SARS) suggests that the virus is the product of a long and private evolutionary history.

Since emerging from southern China in February, SARS has struck at least 4,000 people worldwide and killed more than 200. Disease researchers have launched a massive effort to understand the pathogen and control the epidemic.

On April 16, European scientists announced that they had demonstrated that the agent responsible for SARS is a coronavirus never detected before the cur-

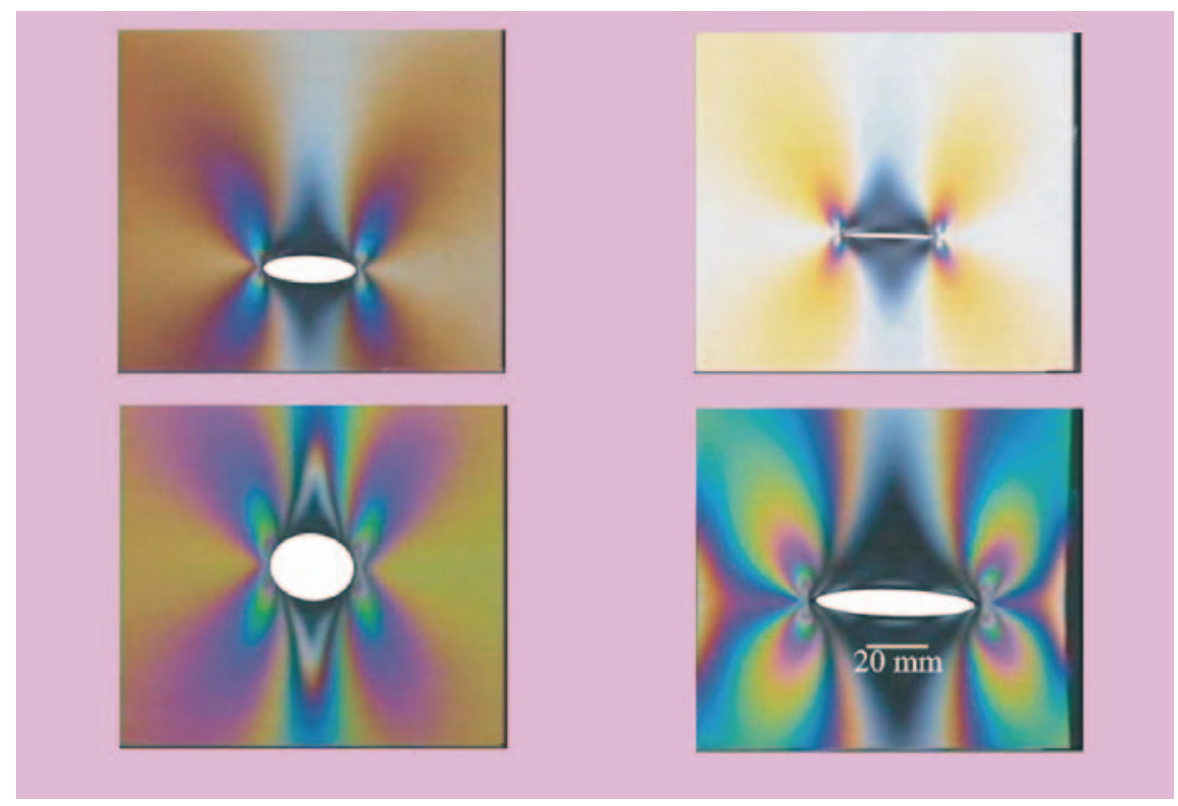

TENSE SCENE In the polymer PVB (left), a horizontal crack transforms into a hole (white). In a similarly elastic but weaker polymer (right), the crack elongates. Colors indicate stress levels. rent outbreak. In experiments on monkeys at the Erasmus Medical Center in Rotterdam, the Netherlands, researchers showed that the new coronavirus alone can cause SARS. Earlier in the outbreak, a member of a separate viral family was also a suspect (SN: 3/29/O3, p. 198).

On April 12, researchers at the British Columbia Cancer Research Centre in Vancouver reported that they had completely sequenced the virus' genome. Scientists in the United States presented nearly identical findings on April 14. Chinese researchers also sequenced the SARS virus and found that some samples differ considerably from those decoded in North America, which suggests that the virus mutates rapidly.

Comparisons among the newly sequenced genome and other coronaviruses' genomes indicate that SARS virus doesn't belong to any of the three known clusters of related coronaviruses. The 10 coronaviruses that infect mammals fall into two clusters, each of which contains one virus that causes colds in people. The third cluster contains two bird pathogens.

Coronaviruses readily swap genetic material with each other in a process known as recombination. This creates new viruses that share some genetic similarities with each parent virus and occasionally have novel capabilities to cause disease or to infect different hosts.

The largest SARS-virus gene, which makes up a whopping two-thirds of the pathogen's genome, appears to be distantly related to the corresponding gene in a mouse coronavirus. Another region of the SARS genome shares a few similarities with the avian coronaviruses.

Those observations suggest that recombination may have given rise long ago to an ancestor of SARS virus, says Michael M.C. Lai, a virologist at the University of Southern California in Los Angeles. If recombination had occurred more recently, the genetic similarities would be more striking, he says.

By itself, Lai notes, "recombination was not responsible for [SARS virus' recent] emergence as a human pathogen."

The SARS virus may have long had the capacity to infect people but only recently encountered conditions that facilitated its spread, says virologist Shinji Makino of the University of Texas Medical Branch in Galveston. Alternatively, it may have derived from one or more unidentified animal coronaviruses that only recently mutated or recombined to create a human pathogen, he says.

The new pathogen "is very likely from a wild animal," argues Lai. Future investigations in the region of China that seems to harbor the virus might eventually turn up the mysterious animal host, he says. - B. HARDER 